\title{
Influence of gravity on a granular Maxwell's demon experiment
}

\author{
N. Isert, C.C. Maaß, and C.M. Aegerter ${ }^{2}$ \\ Department of Physics, Konstanz University, P.O. Box 688, 78457 Konstanz, Germany
}

\begin{abstract}
In the usual description of the granular Maxwell's demon experiment, where phase separation occurs due to an instability in the densities, the control parameter scales linearly with gravity. In this paper we investigate this scaling experimentally using the properties of diamagnetic particles in strong magnetic-field gradients to reduce and even balance gravitation. We find that phase separation occurs even at vanishingly small gravitational accelerations as is predicted by other theories. This is due to the fact that granular samples tend to form clusters as a result of the inelasticity of the particle collisions. Combining the heat balance of the driven granular gas with the cooling rate and thus the appearance of clustering, we are able to describe the crossover between the limiting cases.
\end{abstract}

PACS. 45.70.-n Granular systems - 05.90.+m Other topics in statistical physics, thermodynamics, and nonlinear dynamical systems $-51.10 .+\mathrm{y}$ Kinetic and transport theory of gases

\section{Introduction}

One of the fundamental interests in granular materials is its seemingly counter-intuitive behaviour due to the fact that granular systems are inherently driven [1]. This means that simple interpretations of the second law of thermodynamics fall short of observation, a poignant illustration of which is the so-called Maxwell's demon experiment. In this demonstration experiment, initially devel oped for physics teaching [2], a box is split into two halves by a wall with a slit at a certain height $h$, filled with $N$ granular particles and driven/shaken at a set frequency $f$. When the driving is done slowly, the particles in the box collect in one of the two halves, i.e. the granular system has separated into a low- and a high-density phase. This is particularly surprising since it seems to indicate that order can be created from a random driving of particles. Taking into account the inelasticity of the collision between particles, the effect can be described theoretically [3], which quantitatively predicts the observed instability. A similar approach can also lead to ratcheting behaviour in a granular gas, when many compartments are arranged next to each other [4].

The theory [3] adopts kinetic gas theory [5] to derive a conditional equation for the transition from the state of equal distribution to the phase-separated one. It does so by assuming that all energy inserted into the system per driving cycle, i.e. the heating rate, must be balanced by the dissipation rate due to inelastic collisions. From this, one can then derive a constant granular tempera-

\footnotetext{
${ }^{a}$ e-mail: christof aegerterQuni-konstanz.de
}

ture $T_{h}$ at height $h$ above the bottom boundary. Note that in this approach it is assumed that the temperature is constant in time, i.e. that the driving is fast on the time scale of the cooling rate inherent in a granular gas [6]. Given such a temperature, a granular version of the Navier-Stokes equation yields a particle density distribution akin to that of an ideal gas, i.e. a barometric height formula $n(z) \propto \exp \left(-g z / T_{z}\right)$. This density distribution and the constant temperature directly give the particle flux $j$ from either side of the box into the other via $j \propto n(z) \sqrt{T_{z}}$. Equating these fluxes on both sides of the divide and looking only at deviations from the mean particle number, gives a condition for phase separation as

$$
2 \varepsilon=\tanh (\mu 2 \varepsilon) .
$$

Here $2 \varepsilon=\left|\left(N_{\text {left }}-N_{\text {right }}\right) / N\right|$ is the order parameter, describing the degree of separation varying from 0 to 1 . In addition, $\mu \propto g h / T_{h}$ is the control parameter, where naturally the ratio of dissipative to driving forces enter via the determination of $T_{h}$. Equation (1) is very similar to the definition of the Langevin function in the description of paramagnets and describes a second-order phase transition between the ordered and disordered state. This can also be described as a pitchfork bifurcation, where there is only one stable solution $\varepsilon=0$ for $\mu$ smaller than its critical value $\mu_{\text {crit }}=1$, while two stable solutions $\varepsilon \neq 0$ evolve if $\mu>\mu_{\text {crit }}$. Due to the fact that the control parameter is given by the balance of driving and dissipative forces, it strongly depends on the way the system is heated as well as on the height dependence of the particle density distribution. In [3] the system is driven using a saw-tooth 
oscillation at a frequency $f$ with an amplitude $a$. This means that all particles colliding with the bottom boundary will always find it moving upwards at the driving velocity $v_{b}=a f$. Therefore the granular temperature will be directly related to $v_{b}$ by $T_{h} \propto v_{b}^{2} / n$, such that the control parameter will be given by

$$
\mu=\frac{g h \sigma\left(1-\alpha^{2}\right) N / F}{3 a^{2} f^{2}},
$$

where $\sigma$ is the effective area of the particles, $\alpha$ is the coefficient of restitution of the collisions and $N / F$ is the number of particles per area. In the above derivation it was found that there was a critical value of $\mu_{\text {crit }}=1$ above which phase separation occurs. With this scaling of the control parameter, one directly finds that the phase separation occurs at low frequency and that the critical frequency scales with $g^{1 / 2}$. Note that in the experiments described below, the excitation of the system is performed using a square-wave oscillation. This will change this scaling as described in Section 3.

The theory so far has been found to describe the separation process well, both in simulations [3], and in experiment [7]. Subsequently, it has been expanded to describe an arbitrary number of compartments [8], which also describes experiments and simulations to good accuracy. However, all of these results have been obtained under the condition of normal gravity and therefore are not able to test the scaling of the critical frequency with $g$. This is of particular interest since a similar phase separation as that of Maxwell's demon has been predicted even in the absence of external fields such as gravity [9]. Thus there should be a crossover from the theory of Eggers [3] at high $g$ and clustering models such as that of Brey [9], which are valid at zero $g$. However, in such models the numerical values of the critical parameters cannot be reasonably determined for our experimental setup, such that a quantitative comparison at zero $g$ is not possible. Here, we study the crossover between different phase separations by studying Maxwell's demon at a set of varying effective gravitational accelerations $g_{\mathrm{eff}}$ using diamagnetic particles in a strong magnetic-field gradient $[10,11]$. It is possible to achieve levitation for many materials in the strong magnetic fields produced by modern superconducting cryomagnets, such that even the extreme case of microgravity can be tested, where no separation should take place. Furthermore we develop a quantitative description of the diminishing relevance of Eggers' theory at low accelerations due to the time scale inherent in the cooling of a granular gas [6]. This will also give a reasonable description of the instability at zero $g$.

\section{Experimental setup}

A diamagnetic particle acquires an induced moment antiparallel and proportional to the externally applied magnetic field and thereby a potential energy $U \propto B^{2}$. Accordingly, in an inhomogeneous field, the force acting on the material is determined by the product of field and field

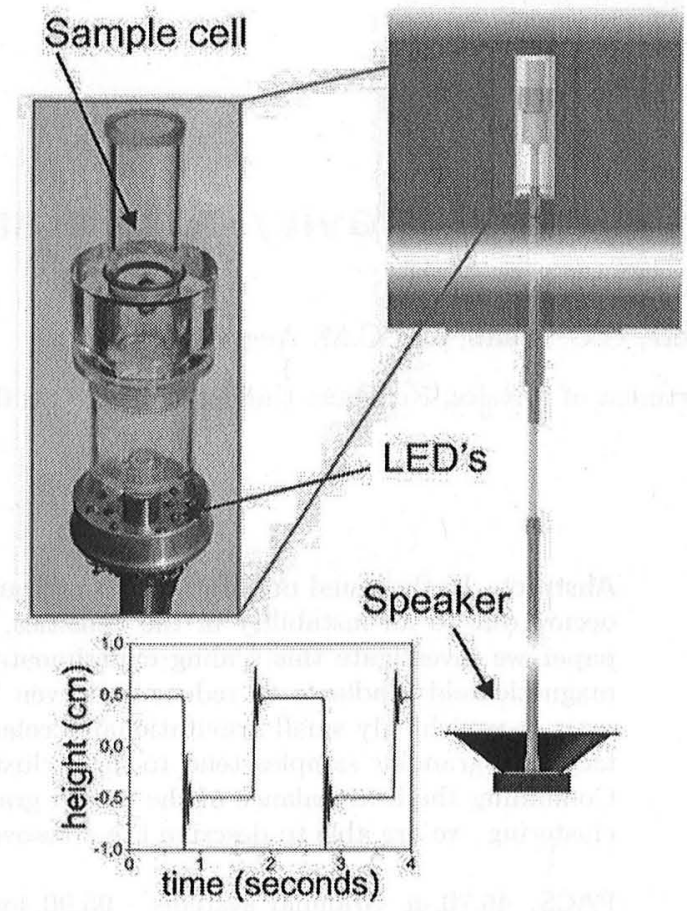

Fig. 1. The setup as used to study the gravity dependence in the Maxwell's demon experiment. The cell has a wall with a horizontal slit inserted and is mounted via a long rod $(60 \mathrm{~cm})$ to the speaker, illumination is from below, the container is observed with a CCD camera from above. The piston position while shaking is indicated in the lower panel showing a square-wave excitation with an amplitude of $1 \mathrm{~cm}$.

gradient, $\boldsymbol{F} \propto \boldsymbol{B} \cdot \nabla \boldsymbol{B}$. The total force acting on the particle above a strong magnetic field thus is the sum of the gravitational force $-m \boldsymbol{g}$ and the diamagnetic repulsion that is proportional to $\boldsymbol{B} \cdot \nabla \boldsymbol{B}$. The effective gravitational acceleration is thus given by

$$
g_{\mathrm{eff}}=g-\frac{\chi}{\mu_{0} \rho} B \cdot \partial_{z} B,
$$

in the direction of the applied field. Radially, the dipolar nature of the field implies a radial force pointing inward, such that the particle can be held in a stable state. If the gravitational and magnetic terms compensate each other in the expression above, we actually have a levitating state comparable to actual weightlessness [11].

The experimental setup [12] is made up of a strong superconducting magnet coil ( $\mathrm{Nb}_{3} \mathrm{Sn}$, Oxford) with a cylindrical bore of $4 \mathrm{~cm}$ in diameter accessible for experiments and a sample container which can be evacuated and is mounted on a loud-speaker via a long connecting rod as is illustrated in Figure 1. A field strength of up to $20 \mathrm{~T}$ can be generated, which corresponds to a maximum $B_{z} \cdot \partial_{z} B$ of approximately $1800 \mathrm{~T}^{2} / \mathrm{m}$. The sample container is made of a closed glass cylinder with an inner diameter of $1.4 \mathrm{~cm}$ split into two parts by a plexi-glass wall, which has a horizontal slit at the height of $1 \mathrm{~cm}$. In order to have a strong effect of diamagnetism, the granular particles 

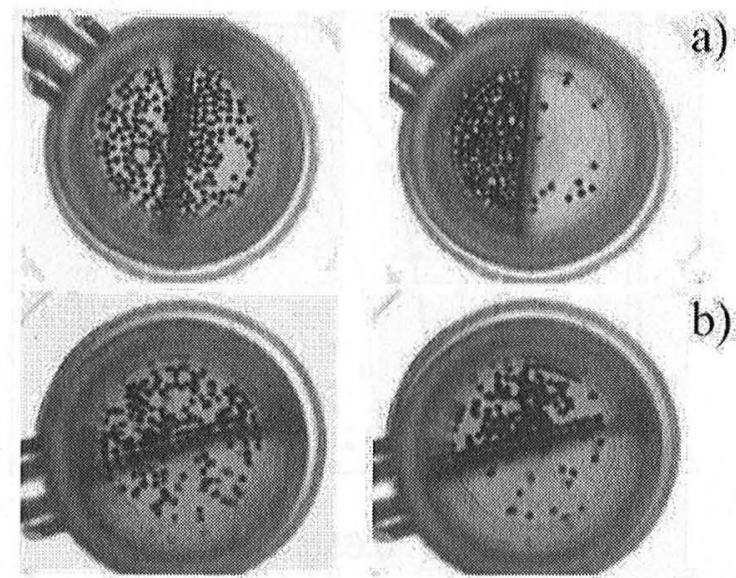

Fig. 2. a) shows a snapshot of the first (left) and last (right) picture of a videotaped Maxwell's demon experiment at $0 \mathrm{~T}$ using 230 bismuth particles. b) shows an early (left) and late (right) snapshot of the same sample in microgravity while the driving is on. There is still some degree of separation.

are made from bismuth due to its high susceptibility of $\chi=165 \cdot 10^{-6}$. The container is filled with 230 bismuth particles with an approximate diameter of $\delta \approx 700 \mu \mathrm{m}$ sorted by weight to have a polydispersity of less than $5 \%$. In our magnet, bismuth requires a field of $13.7 \mathrm{~T}$ (with $\left.B_{z} \cdot \partial_{z} B=730 \mathrm{~T}^{2} / \mathrm{m}\right)$ to levitate.

The sample is illuminated from below using a set of LEDs (visible in Fig. 1), while the dynamics is observed from above using a lipstick CCD camera capable of recording 120 frames per second at a resolution of $640 \times 480$ pixels. The excitation of the sample is obtained by the connection of the container to a loud-speaker, which is driven with a square-wave oscillation. This is because the speaker used here is no longer linear enough to give a good response at the frequencies necessary for a saw-tooth excitation. The amplitude of the driving is fixed to $0.5 \mathrm{~cm}$ in all experiments discussed below.

From the video-recording of the granular container, we are able to obtain the density of particles in the different halves. This is done by either counting the number of particles at the end when particles are at rest or by the average image intensity when the particles are moving too fast to be discerned. We thus experimentally obtain the order parameter $\varepsilon=\left|\left(\frac{N_{\text {side }}}{N_{\text {tota }}}-\frac{1}{2}\right)\right|$ as a function of time. Here we will only discuss the stationary state at the end, as we are interested in the phase separation and not its dynamics. Therefore only the particles in the last frame in the video are studied. The average image intensity is applied to the last 10 frames to gain better statistics. This is only valid if there is no stacking of particles, i.e. if the number of particles is low enough. Snapshots of the first and last frame of a run with a frequency below the transition frequency $f_{\text {crit }}$ are shown in Figure 2 for a sample in normal gravity as well as a sample with levitating particles.

In a typical run, the sample in the container is shaken for a period of $180 \mathrm{~s}$ each at a number of frequencies, while the whole process is being observed from above. A series of frequencies is chosen with smaller intervals close to the transition point $f_{\text {crit }}$ for a more accurate determination of the transition. The procedure is repeated for different applied magnetic fields in order to study directly the effect of gravitation on the critical point of the transition. Thus we are able to determine the dependence of the transition point on the effective gravitational acceleration.

\section{Theoretical description}

As discussed above, the driving method in our experiments is different from the one considered in the derivation of [3] as well as in previous experiments. However, the nature of the instability as described by the dependence of the order parameter $\varepsilon$ on the control parameter $\mu$ as given in equation (1) remains the same, when the control parameter is rescaled to take into account the different type of driving. To be able to compare the experimentally determined order parameter with theoretical predictions, this rescaling is derived here briefly. The main feature of the derivation is in equating of heat rate $Q$ and dissipation rate $I$, such that the driving mainly determines the granular temperature. The heat rate is determined by the energy density transfer $e$ per collision event of a single particle with the bottom boundary and the number of particles that are hit within the time interval $\tau=1 / f$ of one shaking cycle. In case of a saw-tooth driving the energy density transfer is simply given by $e=\rho a b=\rho v_{b}^{2}$, where $\rho$ is the mass density of a particle, and $b=a / \tau^{2}$ the acceleration of the driving. The number of particles is determined by the particle flux close to the bottom. This leads to a temperature dependence of $T_{h} \propto v_{b}^{2}$ and therefore a control parameter that is given by $\mu \propto g / f^{2}$.

For the case of a square-wave excitation as in our experiment, the acceleration is only obtained in a very short time period $\vartheta$ during which the piston moves. Thus only those particles colliding with the bottom boundary within this fraction $\vartheta$ of the oscillation time period $\tau$ will be able to pick up energy. For the energy density transfer per collision one may thus write $e=\rho a b=\rho \frac{a^{2}}{\vartheta^{2}}$. The heat rate then is $Q=n(0) \cdot F \cdot a \cdot \frac{\rho a^{2}}{\vartheta^{2} \tau}$, where $F$ is the area of the container. With the dissipation given by collisions between particles, i.e. $I \propto\left(1-\alpha^{2}\right) n^{2} T^{3 / 2}$, where $\alpha$ is the coefficient of restitution, this results in a granular temperature of

$$
T_{h}=(a f)^{2 / 3} \cdot\left(\frac{a^{2} F}{\sigma N\left(1-\alpha^{2}\right) \vartheta^{2}}\right)^{2 / 3},
$$

where $\sigma$ is the effective cross-section of a particle. In the derivation of the instability, this new temperature needs to be inserted into the density profile with height, $n(z) \propto$ $\exp (-g h / T)$. One thus obtains a similar instability as in equation (1) resulting in

$$
2 \varepsilon=\tanh (\tilde{\mu} 2 \varepsilon) .
$$

Again, the control parameter $\tilde{\mu} \propto g h / T$ is determined by the ratio of temperature and barrier. An exact calculation gives for the control parameter

$$
\tilde{\mu}=\frac{h\left(\sigma\left(1-\alpha^{2}\right) \vartheta^{2} N / F\right)^{2 / 3}}{a^{2} 2^{5 / 3}} \cdot \frac{g}{f_{\text {crit }}^{2 / 3}} .
$$




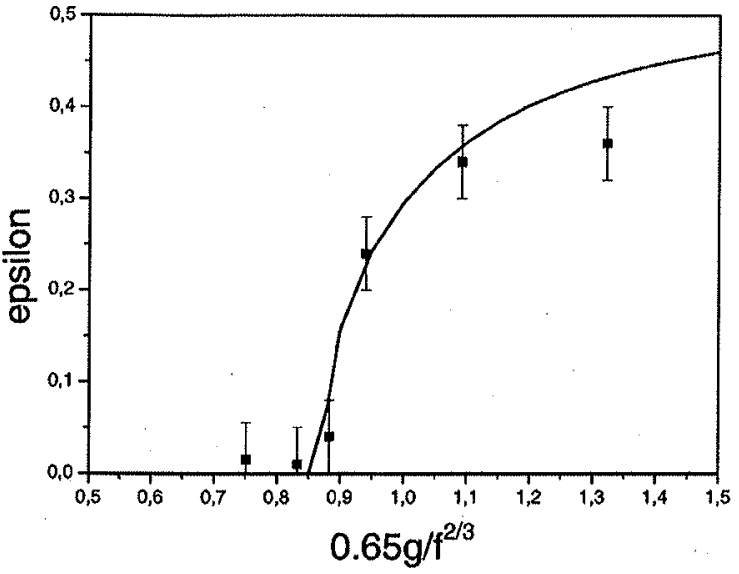

Fig. 3. Bifurcation diagram in the case of an applied field of $10 \mathrm{~T}$. This corresponds to an effective gravitational acceleration of $4 \mathrm{~m} / \mathrm{s}^{2}$. The data were obtained from a series of separation experiments as discussed in Section 2. The critical point is noticeably displaced from its predicted value of 1 . The line is a Langevin function with a critical point at $\mu=0.9$ describing the data within the errors.

As can be seen from equation (5), this control parameter has the critical value of $\tilde{\mu}_{\text {crit }}=1$. The rescaled control parameter scales linearly with the gravitational acceleration as was the case previously but differently with the driving frequency. All the constants entering the prefactor can be determined independently in the experiment, such that this is known without adjustable parameters. In particular, the time $\vartheta$ over which the piston moves is found to be independent of the driving frequency. For the case of the experiments discussed below, we find a control parameter $\tilde{\mu}=0.65 \mathrm{~g} / \mathrm{f}^{2 / 3}$, with $g$ in units of $\mathrm{m} / \mathrm{s}^{2}$ and $f$ in $\mathrm{Hz}$. Therefore the bifurcation can be predicted theoretically and compared to the experiment. An example of this can be seen in Figure 3 and describes the data reasonably well.

\section{Results}

As an illustration of the pitchfork bifurcation nature of the instability, we show the dependence of the order parameter $\varepsilon$ on $\tilde{\mu}$ in Figure 3 . These measurements were obtained at an applied field of $10 \mathrm{~T}$, which corresponds to an effective gravitational acceleration of $4 \mathrm{~m} / \mathrm{s}^{2}$. The data follow the prediction of the theory, i.e. a Langevin function, reasonably well, as can be seen by comparing the data to the full line in the figure. Note, however, that here we have used a critical value of $\tilde{\mu}_{\text {crit }}=0.9$ to describe the data, whereas a value of unity would be predicted theoretically. This indicates that at such lower values of the effective gravitational acceleration, the theory still predicts the nature of the instability but quantitatively disagrees with the experiment. Thus the assumptions behind the theory start to be violated at effective gravitational accelerations comparable to the experiment shown in Figure 3. In comparison, the data at normal gravity follow the prediction

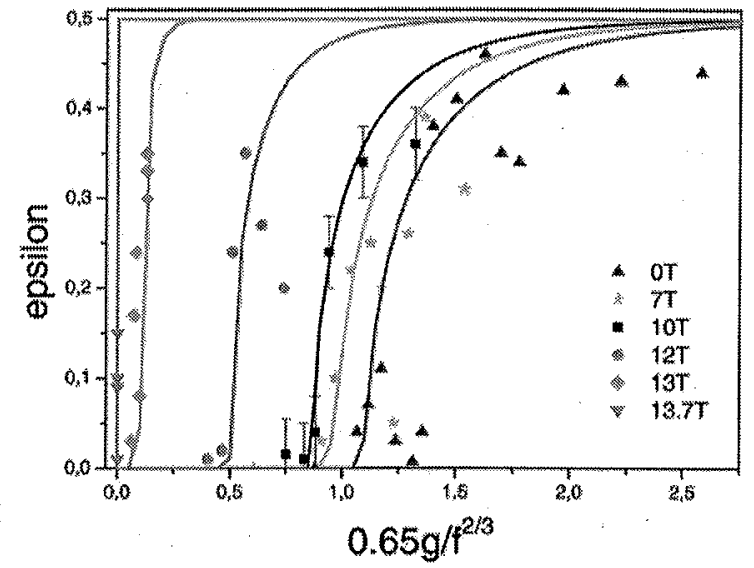

Fig. 4. (Color online) All bifurcations plotted into one graph, scaled with the effective gravitational acceleration. As can be seen, the agreement with the theoretical predictions decreases with decreasing effective acceleration. The critical point for the transition is shifted to lower values than would be expected. This corresponds to a higher critical frequency as can also be seen in Figure 5. Most importantly, there is still a frequency-dependent separation taking place at microgravity. This cannot be resolved here since $g$ vanishes and all frequencies at levitation are plotted on the same line.

with a critical control parameter of $\tilde{\mu}_{\text {crit }}=1$ (see Fig. 4), such that we can see that the validity of the assumptions indeed depends on $g_{\text {eff }}$. The most blatant disagreement of the above theory and our experiment is however indicated in Figure 2, where it can be seen that phase separation also occurs in a levitated sample. In this case, even the shape of the bifurcation is however no longer properly described by the theory. Thus we observe a gradual crossover from data, which are well described by the bifurcation theory outlined above to a qualitative difference of a phase separation where none should occur according to theory. This is summarized in Figure 4, where the different experiments for all effective gravitational accelerations are shown, scaled to their respective $g_{\text {eff }}$. The figure contains data points for bifurcations at effective $g$ 's of $9.8 \mathrm{~m} / \mathrm{s}^{2}(0 \mathrm{~T}), 7 \mathrm{~m} / \mathrm{s}^{2}(7 \mathrm{~T}), 4 \mathrm{~m} / \mathrm{s}^{2}(10 \mathrm{~T}), 1.8 \mathrm{~m} / \mathrm{s}^{2}(12 \mathrm{~T})$, $0.2 \mathrm{~m} / \mathrm{s}^{2}(13 \mathrm{~T})$ and $0 \mathrm{~m} / \mathrm{s}^{2}(13.7 \mathrm{~T})$. The deviations from the prediction $\tilde{\mu}_{\text {crit }}=1$ increase with decreasing $g_{\text {eff }}$ and are highly significant for $g_{\text {eff }}<3 \mathrm{~m} / \mathrm{s}^{2}$. In fact, at such low effective accelerations, the bifurcation diagrams no longer follow a Langevin function in the control parameter $\tilde{\mu}$. Thus the data indicate that there is a shift in the critical value of the instability and even the nature of the instability seems to be changed at very low gravitational accelerations.

The data can also be summarized via their critical frequency. The scaling of the control parameter with $g$ directly gives a dependence of $f_{\text {crit }} \propto g^{3 / 2}$, which is shown by the solid line in Figure 5. As can be seen in that figure, the critical frequencies are in reasonable agreement with the prediction for high values of the effective gravitational acceleration (Eq. (6)) indicated by the solid, red 


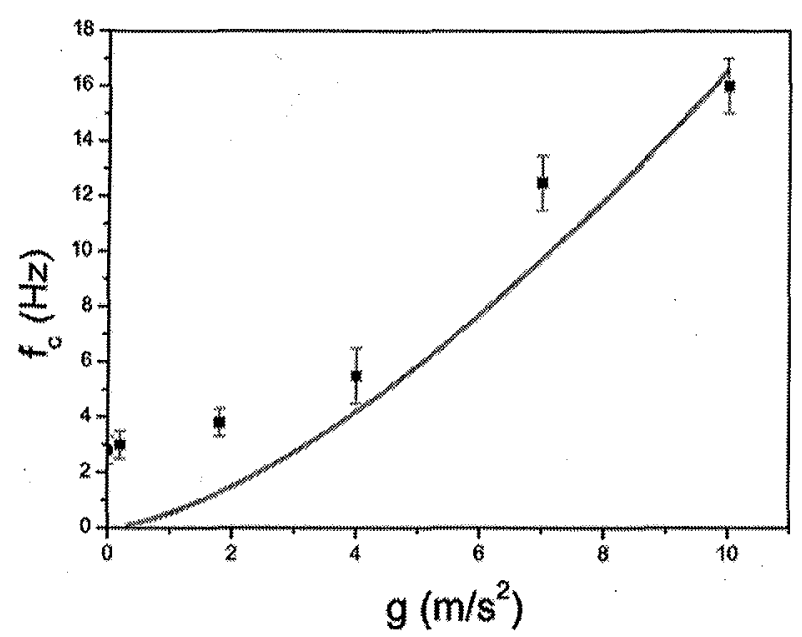

Fig. 5. (Color online) Dots: data point of the critical frequency over the effective gravitational acceleration as obtained from the transition points in the measured bifurcations. Red curve: theoretical prediction of the ratio of critical frequency to gravitational acceleration. There is good agreement for normal gravitation while the deviations become more pronounced for values of $g_{\text {off }}$ below about $4 \mathrm{~m} / \mathrm{s}^{2}$.

line, but start to deviate at values around $g_{\text {eff }} \simeq 4 \mathrm{~m} / \mathrm{s}^{2}$. This corresponds to critical frequencies of roughly 3 to $4 \mathrm{~Hz}$. In the derivation above, it was assumed that on the time scale of the driving, the dynamics of the gas should be homogeneous. However, it was shown recently that in a levitated sample, depending on the particle density and the mean speed, there is a time scale of the order of a second on which the granular gas cools and therefore forms clusters [12]. This means that on this time scale, the Haff time $\tau_{H}^{-1}=n\left(1-\alpha^{2}\right) \sigma \sqrt{T} / 2$, the assumption of homogeneity is no longer valid. Given the temperature of the gas derived above (Eq. (4)), this time scale can be calculated for our experiments. Equating the Haff rate with the driving frequency gives an estimate of the critical frequency at which clustering becomes important due to the driving mechanism and therefore separation should occur in the absence of gravity. One obtains

$$
f_{c}=\frac{\sigma\left(1-\alpha^{2}\right) N}{\vartheta F} \cdot\left(\frac{a}{2 H}\right)^{3 / 2}
$$

where $H$ is the effective height filled by the granular gas. This height is determined by the sample cell height as well as the depth of the levitating potential. Both of these scales are around $3 \mathrm{~cm}$. Thus using such a reasonable value of $H \simeq 3 \mathrm{~cm}$, we obtain a critical frequency of $f_{c} \simeq 3.5 \mathrm{~Hz}$ as was shown in the experiment. It is interesting to note that the critical parameter here directly scales with the density as is the case in the theory of Brey [9] for a realization of Maxwell's demon in the absence of gravity. Similarly, this argument can be used to obtain an estimate of the effective acceleration at which Eggers' theory should break down, which is the case when the critical frequency from equation (6) is equal to that of equation (7).
With this, one obtains a value of $g_{c}=\frac{2^{2 / 3} a^{3}}{h H \vartheta^{2}} \simeq 4(1) \mathrm{m} / \mathrm{s}^{2}$ in good agreement with the experiment (see Fig. 5).

\section{Conclusions}

In conclusion, we have shown that a phase separation is achieved in a granular Maxwell's demon experiment even in the absence of a gravitational acceleration. When changing the effective gravitation by applying different magnetic fields to diamagnetic granular samples, one can see that at high values of $g$ the established theory can describe the data reasonably well, but starts to disagree at accelerations around $4 \mathrm{~m} / \mathrm{s}^{2}$. As discussed in the introduction, the derivation of the theory assumes a homogeneous heating mechanism that is fast compared to the heat conduction in the granular gas and to the ensuing homogeneity of the density. When taking into account the effect of clustering in a granular gas, a phase separation can be obtained even in the absence of gravity [9]. As has recently been shown [12], when the effect of gravity is reduced, the inherent cooling process due to the collisions can be observed. This leads to an inhomogeneity in the density, which naturally gives rise to a clustering. Thus the time scale of cooling can be treated as a relevant time scale on which phase separation can occur. The scaling of the critical parameter in this case is similar to that derived by Brey [9], but in contrast to this complete theory, we can estimate the coefficients entering in the cooling time, such that a comparison to the experiment becomes possible. As the cooling time scale becomes longer as $g$ is reduced, it will become comparable with the critical driving frequency for phase separation at some effective acceleration $g_{c}$. For the values of our experiment, this happens at an effective gravity of roughly $4 \mathrm{~m} / \mathrm{s}^{2}$. As can be seen from the data in Figure 5, this is the region of effective gravity where the experimental deviations from the prediction start to become significant. Therefore we would conclude that the inherent cooling and therefore clustering in the granular gas allows for a phase separation even in the absence of gravity, as we also observe experimentally.

This work was supported by the Deutsche Forschungsgemeinschaft via the IRTG 667 as well as the Landesstiftung BadenWürttemberg. Furthermore, we would like to thank Georg Maret for fruitful discussions.

\section{References}

1. H.M. Jaeger, S.R. Nagel, R.P. Behringer, Rev. Mod. Phys. 68, 1259 (1996).

2. H.J. Schlichting, V. Nordmeier, Math. Naturwiss. Unterr. 49, 323 (1996).

3. J. Eggers, Phys. Rev. Lett. 83, 5322 (1999)

4. D. van der Meer, P. Reimann, K. van der Weele, D. Lohse, Phys. Rev. Lett. 92, 184301 (2004).

5. J.W. Dufty, Phys. Rev. A 13, 2299 (1976).

6. P.K. Haff, J. Fluid Mech. 134, 401 (1983).

7. K. van der Weele, D. van der Meer, M. Versluis, D. Lohse, Europhys. Lett. 53, 328 (2001). 
8. D. van der Meer, K. van der Weele, D. Lohse, Phys. Rev. E 63, 061304 (2001).

9. J.J. Brey, F. Moreno, R. Garcya-Rojo, M.J. Ruiz-Montero, Phys. Rev. E 65, 011305 (2001).
10. W. Braunbek, Z. Phys. 121, 764 (1939).

11. M.V. Berry, A.K. Geim, Eur. J. Phys. 18, 307 (1997).

12. C.C. Maass, N. Isert, G. Maret, C.M. Aegerter, Phys. Rev. Lett. 100, 248001 (2008). 